\title{
ZU EINIGEN AEGYPTIACA AUS DEM NATIONALMUSEUM FÜR RUMÄNISCHE GESCHICHTE, BUKAREST (RUMÄNIEN)
}

Robert Kuhn

\section{ABOUT SOME AEGYPTIACA FROM THE NATIONAL HISTORY MUSEUM, BUCHAREST (ROMANIA)}

In the following article we will publish six predynastic objects of pottery given to the Museum as a donation by Mihail C. Sutzu. All items are made of Nile clay and date back to the stages of Naqada I-II. It looks like as they could all belong to the same tomb inventory. Just one pot, belonging to Petrie's P-Ware is a bit younger in date (Naqada II/III). The description and dating as well as a try of interpretation follows a short critical summary of Petrie's seriation method developed at the beginning of the $19^{\text {th }}$ century. The second part will deal with the presentation of a funerary cone of the Highpriest of Amun, $M n-h p r-R^{r}-s n b$, dating back to the $18^{\text {th }}$ Dynasty.

Keywords: Egyptian Pottery; Egyptian Predynastic Period; Formative Phase; Black-Topped-Ware; Red-Polished Ware; Naqada; Petrie; Sutzu; Bolliac; Menkheperrasonb; Funerary Cone; Egyptian Mummy

Das Nationalmuseum in Bukarest' besitzt eine umfangreiche Abteilung archäologischer Schätze unter denen sich auch einige wenige Aegyptiaca befinden. Die zu besprechenden Stiicke stammen aus der Privatsammlung von Mihail C. Sutzu (1841-1933), einem politisch engagierten Sammler und Numismaten, der u.a. auch Direktor der Rumänischen Nationalbank war. 1870 unternahın der passionierte Sammler von Antiquitäten eine Studienreise nach Ägypten, die ihn bis zum zweiten Katarakt führte. Er tätigte hierbei diverse Ankäufe ${ }^{2}$. Die meisten der Objekte seiner Sammlung ent-stammen aber vorwiegend lokalen Grabungen wie beispielsweise Tomis. Nach dem I. Weltkrieg vermachte er seine Privatkollektion der Rumänischen Akademie der Wissen-schaften, deren Mitglied er seit 1909 war. Ins-gesamt umfasst sie 3105 Numismatika, 241 Bleisiegel, 360 Skulpturen, verschiedene Terra-kotten,

\footnotetext{
1 Folgenden Personen sei für die Bereitstellung des zu publizierenden Materials. sowie die freundliche Auf-nahme und Unterstützung gedankt: Herrn Dr. Muşeţeanu. Herrn Dr. Trohani, Frau Velicu. M.A., Frau Licuță. Besonderer Dank vor allem für Anmerkungen gilt Frau Böhme, M.A.. Irau Terlelzki. M.A. und Frau Mahn.

? Vgl. Vianu 1992. 131.
}

Keramiken, Bronzen und Bücher ${ }^{3} .1957$ wurde die Sammlung dem Muzeul Naţional de Antichităţi eingegliedert, das kurz darauf schloss. Die ehemals Sutzu gehörenden Objekte wurden 1972 auf verschiedene Institutionen aufgeteilt, etwa das Muzeul Naţional de Istorie a Romaniei, die historische Fakultät der Universität und das Archäologische Institut. Heute befindet sich ein Großteil der Gegenstände in den Depots des Nationalmuseums.

Nähere Informationen zum Ankauf und der Provenienz der einzelnen Objekte sind nicht bekannt. Sie dürften aber auf dem Antikenmarkt während seiner Ägyptenreise angekauft worden sein. Es handelt sich vorwiegend um Keramikobjekte, die vermutlich einem bzw. zwei prädynastischen Gräbern entstammen. Hinzu kommt ein aus gebranntem Ton bestehender Grabkegel mit Stempelsiegel.

Neben den keramischen Objekten beherbergt die Sammlung des Historischen Museums in Bukarest auch Mumien. Im Depot des Museums befinden sich drei Mumien und Reste einer vierten, die von dem Poeten und

${ }^{3}$ Vianu 1992, 136-137. 
Archäologen Cezar Bolliac (1813-1881) 1872 dem Muzeul Naţional de Antichităţi mit weiteren Objekten seiner Privatsammlung ver-macht wurden ${ }^{4}$. Neben zwei Kindermumien be-findet sich hierunter auch die Mumie des Bes (Fig. 3,3), dessen Kartonage heute in der Fakultät für Geschichtswissenschaften der Uni-versität Bukarest aufbewahrt wird ${ }^{5}$. Aufgrund stilistischer Vergleiche datierte Cihó die Mumie mitsamt Kartonage völlig zu Recht in die 22.-23. Dyn. Die restlichen drei Mumien können aufgrund fehlender Sarkophage und Särge nicht mit Sicherheit datiert werden.

\section{Prädynastische KeramiK}

Inv.-Nr. 121488 - alt: E. $76(\mathrm{Vgl}$. Fig. 1,1 und Fig. 2,4)

Maße: H.: 12,5 cm; Md.-Dm.: 19,0-19,4 cm; B.Dm.: 7,5-8,0 cm; Wandstärke: $0,7-1,1 \mathrm{~cm}$

\section{Zustand: komplett erhalten}

Form: leicht konische Schüssel mit direktem Rand

Bearbeitung außen: Das Gefäß wurde handaufgebaut und die Oberfläche vertikal geglättet sowie glänzend poliert. Der obere Rand ist im Brennvorgang schwarz geschmaucht worden und glänzt aufgrund der Politur metallen (bis max. 4,1 $\mathrm{cm}$ ab Md.). Der Wackelboden ist un-poliert und weitgehend ungeglättet belassen worden, weist aber ein dünnes, horizontal um den gesamten Körper laufendes rotbraunes Zier-band auf, das sich $1 \mathrm{~cm}$ über dem Boden befindet. Die Oberfläche ist kaum windver-schliffen. Die Oberflächenfarbe unterhalb des schwarz geschmauchten Randes ist weitgehend rötlichbraun.

Bearbeitung innen: Die Bearbeitungsspuren innen sind gut sichtbar. Das Gefäß ist hand-aufgebaut und ebenso handgeglättet, deutliche Spatelspuren sind nicht sichtbar. Der Rand ist hervorragend geglättet, dagegen sind Körper und Boden roh

\footnotetext{
${ }^{4}$ Vgl. Potra 1944, 243. Ebenso: Ionitā 2004, 195-2I5.

${ }^{5}$ Die Kartonage wurde bereits von M. Cihó 1984, 83-87 publiziert. Das Objekt trägt dic Inv.-Nr. E.192. Ebenso: Cihó et al. 1988, 25. Hier wird allerdings eine andere Inv.Nr. genannt: E.40 UB. Unter der Rubrik ..menschliche Mumien" werden allerdings die vier im MNIR belindlichen Objekte nicht aufgeführt. Vgl. 31-33.
}

belassen. Das komplette Innere des Gefäßes ist aufgrund des Brennvorgangs schwarz gehalten.

Magerung: Nilton A; vorwiegend mineralisch (Kalkausbrüche von bis zu $0,2 \mathrm{~cm}$ ) - es sind keine Häckselspuren auf der Oberfläche erkennbar.

Datierung: Naqada Ic-Naqada IIa (ca. 3700/600 v. Chr.), Petrie S.D. 31-34 $4^{6}$ Korpusnr.: B $18 \mathrm{~g}^{7}$

Inv.-Nr. 121489 - alt: E. $77(\mathrm{Vgl}$. Taf. 1.1 und Fig. 2,2)

Maße: H.: 21,5 cm; Schulter-B.: 13,4 cm; Md.Dm.: 14,2-14,5 cm; B.-Dm: 6,7-6,9 cm Wandstärke: $0,4-0,7 \mathrm{~cm}$

Zustand: nahezu komplett, kleine Ausbrüche und -platzer am Rand

Form: leicht konisch nach oben auslaufendes Hochgefäß mit Wackelboden

Bearbeitung außen: Das Gefäß ist handaufgebaut, außen gut geglättet und glänzend poliert. Vom Rand bis knapp unterhalb der Schulter ist die Oberfläche geschmaucht und glänzt stark metallen. Der Wackelboden ist relativ breit und unpoliert belassen Die Oberfläche ist punktuell teils stark windverschliffen und zeigt noch durch das leicht wellenförmige Profil den Handaufbau. Die Oberflächenfarbe ist rötlich-braun im Bodenbereich, hell-gelblich-rot dort, wo keine Politur erfolgte.

Bearbeitung innen: Das Gefäß ist in Wulsttechnik handaufgebaut worden, die einzelnen Wülste sind gut zu erkennen und mäßig bis leicht überstrichen und geglättet. Teilweise sind noch horizontal verlaufende Fingerverstrich-spuren und vertikal angesetzte Spatelansätze er-kennbar. Das

\footnotetext{
${ }^{6}$ Im Folgenden werde ich die Staffeldaten/Sequence-Dates (S.D.) und die jeweilige Korpusnummer für die einzelnen Gefäße benutzen. Als Standardwerke hierfür dienen: Petrie 1899, 295-301 und Petrie 1901, be-sonders pl. II-III. Der Korpus selbst wurde erneut in Petrie 1921 vorgestellt. Eine Überarbeitung des Systems erfolgte durch Kaiser und Hendrickx, die sich auch kritisch mit dem System des britischen Archäologen auseinandersetzten (s.u.). Vgl. Kaiser 1957, 69-77 und Hendrickx 1999, 13-81. Eine neue Überarbeitung der frühen Chronologie von Naqada und der Typologie ist bereits von Buchez 2007. Für Abydos: Hartmann 2008, 103-104.

${ }^{7}$ Die Zuweisung ist etwas schwierig, da Petrie 1921, pl. II in seinem Korpus lediglich Formen aufgelistet hat. die unser Zierband überhalb des Bodens nicht auf-weisen. Von der Form her finden wir das Objekt aus Bukarest in Petries Nr. $18 \mathrm{~g}$ wieder.
} 
komplette Innere ist durch reduzierenden Brand schwarz.

Magerung: Nilton A; mineralisch als auch organisch gemagert. Auf der Oberfläche sind teils durch den Windschliff gezeigt, viele Kalk-partikel von bis zu 0,1-0,2 cm Größe erkennbar, zudem enthält der Ton wenig bis mäßig Glimmer. An organischen Magerungspartikeln sind wenige Häckselausbrennungen auf der Oberfläche zu vermerken.

Datierung: Naqada Ib-Naqada IIb (ca. 3800 3500 v. Chr.); Petrie S.D. 30-55; Korpus-Nr.: B $25 b$

Inv.-Nr. 121490 - alt: E. 78 Vgl. Fig. 1,1 und Fig. 2.1)

Maße: H.: 21,8 cm; Schulter-B.: 10,2 cm; Md.Dm.: 10,7-11,1 cm; Bd.-Dm.: 5,3 cm; Wandstärke: $0,5-0,6 \mathrm{~cm}$

Zustand: nahezu komplett, nur kleine Ausbrüche im Randbereich

Form: konisches Hochgefäß mit schwach ausgezogenem Rand und Wackelboden

Bearbeitung außen: Das Gefäß wurde handaufgebaut aber innen und außen sehr gut geglättet. Die Oberfläche ist zudem sehr gut poliert, punktuell existieren windverschliffene Stellen. Der Rand ist schwarz geschmaucht und glänzt metallen (bis $\max .4 .9 \mathrm{~cm}$ ab Md.). Die Glättungs- und Polierspuren verlaufen senkrecht. Der Wackelboden ist unpoliert und nur leicht geglättet belassen worden. Vor allem im Bereich des Randes befinden sich Spuren von Windschliff. Die Farbe der Oberfläche unterhalb des schwarz geschmauchten Randes ist rötlich-braun.

Bearbeitung innen: Der Mündungsbereich ist teils glänzend poliert bis zu einer Höhe von max. 4,6 $\mathrm{cm}$ ab der Mündung. Der Gefäßkörper ist fingerverstrichen und weist senkrecht verlaufende bis zu $3.1 \mathrm{~cm}$ lange Spatelansatzspuren auf.

Magerung: Nilton A; vorwiegend mineralisch gemagert, Kalkstiickchen sind an der Oberfläche bis zu einer Größe von 0,2 cm erkennbar, relativ hoher Anteil an Glimmer; wenige Häckselausbrennungen auf der Oberfläche bis zu 1,2 cm Länge.
Datierung: Naqada Ib-Naqada Ila (ca. 38003600 v. Chr.); Petrie S.D. 33-45; Korpus-Nr.: B 27d

Inv.-Nr. 121491 - alt: E. 80 (Vgl. Fig. 1,1 und Fig. 3,1)

Maße: H.: 27,0 cm; B.-Dm.: 5,8-6,2 cm; Md.Dm.: 14,5-14,8 cm; Wandstärke: 0,7-0,9 cm

Zustand: nahezu komplettes Gefäß, leichte Ausbrüche am Rand

Form: schwach doppelkonisches Gefäß, die Mündung ist leicht nach außen ausgezogen, der Bauch schwingt leicht aus; Wackelboden

Bearbeitung außen: Das Gefäß wurde in Wulsttechnik handaufgebaut, die auch noch an der äußeren Oberfläche gut sichtbar ist. Die Oberfläche ist senkrecht geglättet und hochglänzend poliert worden. Der Rand ist durch den Brennvorgang geschmaucht und schimmert metallenschwarz. Während der obere Rand bis max. $5,3 \mathrm{~cm}$ ab Md. schwarz geschmaucht ist, folgt eine hellbraune Zone bis ca. 8,6 cm ab Md. Erst ab 8,6 $\mathrm{cm}$ ab Md. hat das Gefäß eine nahezu gleichmäßige rot-braune Färbung. Das Besondere an diesem Objekt ist, dass auch der Boden geglättet, wenngleich nicht poliert worden ist.

Bearbeitung innen: Das Innere des Gefäßes ist gut bis sehr gut geglättet, der Boden fingerverstrichen und erst der obere Rand ca. ab der Schulter $(9,2 \mathrm{~cm}$ ab Md.) weniger sorgfältig geglättet. Hier sind nun auch deutliche Fingerverstrichspuren (schräg-horizontal verlaufend), Fingerabdrücke und Spatelansatzspuren (senkrecht verlaufend) sichtbar. Nur der unmittelbare Mündungsbereich (bis $\max .1,7 \mathrm{~cm}$ ab Md.) ist geschmaucht und geschwärzt, ansonsten ist die Farbe im Innern des Gefäßes eher grau-braun.

Magerung: Nilton A; vorwiegend mineralisch gemagert; mäßig Kalk, mäßig-viel Glimmer, wenige Häckselausbrennungen und -spuren sind auf der Oberfläche erkennbar.

Datierung: Naqada Ib-Naqada Ilb (ca. 38003500 v. Chr.); Petrie S.D.: 36-50; Korpus-Nr.: B $25 \mathrm{~h} / 25 \mathrm{n}$

Inv.-Nr. 121493 - alt: - (Vgl. Fig. I,2 und Fig. 3,2) 
Maße: H.: 23,8 cm; B.-Dm.: 4,3-4,5 cm; Md.Dm.: 9,2-9,4 cm; Wandstärke: 0,7-0,9 cm

Zustand: zerscherbtes Gefäß, das geklebt und ergänzt wurde (7 Scherben), ein Originalprofil ist noch erhalten; der obere Rand ist leicht abgerieben.

Form: Kleine Flasche mit leicht ausgezogener Randlippe und leicht konkav einziehendem Rand; die Schulter zieht bei ca. 2/3 des Gefäßes aus; schmaler Wackelboden

Bearbeitung außsen: Die Flasche ist handaufgebaut, die Oberfläche aber gut geglättet und verstrichen, so dass kaum noch Aufbauspuren erkennbar sind. Die Glättungs- und Politurspuren verlaufen senkrecht. Stellenweise - teils auf einer ganzen Seite - ist die Oberfläche nahezu komplett windverschliffen. Der Hals ist hori-zontal strichpoliert, wobei der restliche Körper vertikal strichpoliert wurde. Der Halsumbruch wurde vermullich mit einem Formholz oder Spachtel herausgearbeitet. Möglicherweise wurde das Gefäß auf einer langsam drehenden Töpferscheibe nachgedreht. Die Färbung des Gefäßes ist rötlich-braun.

Bearbeitung innen: Die Wulstansätze sprechen für einen Handaufbau in Wulsttechnik, die teils mit Fingerverstrichspuren geglättet wurden. Die Fingerverstrichspuren verlaufen horizontal, die Spatelansatzspuren eher senkrecht mit horizontalen Verstrichspuren. Während der innere Körper teils sehr gut geglättet wurde, weisen Hals- und Mündungsbereich auch innen Glättspuren bis max. 2,7 cm ab Md. auf.

Magerung: Nilton; vorwiegend mineralisch mit viel - sehr viel Kalk und wenig bis mäßig Glimmer gemagert; wenige kleine Tonsteine, organische Magerungsbestandteile sind kaum zu konstatieren.

Datierung: Naqada IIa-Naqada IIla (ca. 36003300 v. Chr.); S.D. 42-70; Korpus-Nr.: P 40e

Inv.-Nr. 121495 / alt: E. 85 Vgl. Fig. 1,1 und Fig. 2,3)

Maße: H.: 23,0 cm; Schulter-Dm.: $11,4 \mathrm{~cm}$; Bd.Dm: 4,0 cm; Md.-Dm.: 9,5-9,7 cm; Wandstärke: $0,5-0,7 \mathrm{~cm}$

Zustand: zerscherbt (17 Fragmente), geklebt und ergänzt, ein Originalprofil ist erhalten
Form: Hochgefäß mit schwach ausbiegendem Rand und rundlich-ovaler Md. in der Aufsicht; die Schulter schwingt leicht aus; Wackelboden

Bearbeitung außen: Das Gefäß wurde handaufgebaut und geglättet, sowie hochglänzend poliert. Der Rand ist schwarz geschmaucht und metallen-schwarz glänzend (bis max. $4,3 \mathrm{~cm}$ ab Md.), wobei die restliche Oberflächenfarbe rötlich-braun, teils auch sehr hell ist. Der Wackelboden ist relativ schmal und nicht nachbearbeitet.

Bearbeitung innen: Der Handaufbau in Wulsttechnik, sowie die erfolgte Glättung sind gut sichtbar. Der gesamte innere Körper weist leicht schräg nach oben rechts führende teils bis zu $2 \mathrm{~cm}$ lange Spatelansatzspuren auf. Im Schulter-bereich sind zudem noch leicht Fingertupfen er-kennbar. Einzig der Mündungsbereich wurde gut geglättet und poliert (bis max. 1,3 cm ab Md.).

Magerung: Nilton A; organisch als auch mineralisch, wenige ausgebrannte Häcksel-spuren und Kalkausbrennungen auf der Ober-fläche erkennbar

Datierung: Naqada Ic-Naqada IIb (ca. 36003500 v. Chr.); S.D. 32-43; Petrie B 35a

\section{INTERPRETATION UND EINIGE BEMER-KUNGEN ZUR SERIATION DER NAQADA-WARE DURCH PETRIE}

Die oben besprochenen Gefäße sind allesamt der Stufe Naqada I (ca. 3900-3600 v. Chr.) im Übergang zur Stufe Naqada II (ca. 3600-3300 v. Chr.) zuzuordnen. Auch wenn der Fundkontext und die Provenienz der Objekte nicht bekannt sind, ist dennoch wahrscheinlich, dass es sich bei allen Gefäßen um Grabkeramik handelt. ${ }^{8}$ Sie wurden von Sutzu zu einem Zeit-punkt aufgekauft, als die ägyptische Prähistorie noch gänzlich unerforscht war.

Bereits in der frühen Stufe von Naqada, am Übergang vom Neolithikum zum Chalkolithikum, hat sich eine Kultur im Niltal herausgebildet, die über ein spezialisiertes Töpferhand-

\footnotetext{
${ }^{8}$ Dic Hauptanzahl der Keramik stammt aus Gräbern. aber selbst lür die Decorated-Ware konnten einige Ob-jekte auch im Siedlungskontext nachgewiesen werden. Die sonst praktizierte eindeutige Trennung von Grab- und Siedlungskeramik lässı sich daher nicht mehr aufrechterhalten. Vgl. Friedman 1994, 84-86.
} 
werk verfügte. Charakteristisch ist, dass die Alltagsgefäße, die wir aus den Siedlungen kennen, zumeist grob geschlemmte, schlecht gebrannte Keramik ist. Die evaluierte Ware, die meist mit einer Schmauchung des oberen Randes verziert ist, stammt hingegen aus Gräbern und diente als besondere Beigabe für den Toten. Im Durchschnitt wurden dem Verstorbenen nur wenige Gefäße ins Grab mitgegeben, es existieren freilich aber auch Ausnahmen mit bis zu 80 Gefäßbeigaben?. Über einen besonderen Gefäßinhalt ist uns bisher nichts bekannt und auch die hier besprochenen Exemplare weisen keinerlei Hinweise ihre Nutzung betreffend auf. Möglich ist, dass sie als leere Gefäße, im Sinne einer parspro-toto-Beigabe, ins Grab kamen. Eine weitere Möglichkeit zur Nutzung dieser Gefäße könnte im Zusammenhang mit einem Nahrungs-ritual am Grab während der Bestattung sein. So konnte Hendrickx in der Nekropole von El-Kab auch eine große Anzahl von Gefäßen in der Nähe der einzelnen Grabgruben nachweisen. ${ }^{10}$ Des Weiteren ist zu annotieren, dass viele der bisher gefundenen Objekte durchaus Gebrauchs-spuren aufweisen. Dies ist wohl sicher mit Ritualmalzeiten u.ä. am Grab zu erklären (s.u.).

Die hier besprochenen Gefäße sind nahezu allesamt der von Petrie so genannten B-Ware, der Black-topped-Ware, zuzuordnen. In Ägypten kommen sie in der Badari-Kultur auf und laufen bis zum Ende der Naqada II-Zeit. Nur wenige Exemplare sind auch aus der jüngeren Stufe von Naqada III bekannt." In Ägypten fällt das Verschwinden dieser Ware mit dem Auf-kommen der Wellenhenkelgefäße, der von Petrie so genannten W-Ware zusammen. Letztere Gefäßtypen wurden vorwiegend aus Mergelton gefertigt, so dass sie bis auf $1000^{\circ} \mathrm{C}$ in den Töpferöfen gebrannt werden konnten ${ }^{12}$. Es besteht somit möglicherweise ein Zusammenhang mit den neuen Brenntechniken. Im Sudan hingegen wird die B-Ware noch bis zum Ende der Kerma-Kultur (ca. 1500 v. Chr.) verwendet. In abgewandelten Formen ist die Black-Topped

\footnotetext{
${ }^{9} \mathrm{Vgl}$. Holfman 1980, 116.

${ }^{10} \mathrm{Vgl}$. Hendrickx 2002, 47-54.

$"$ Vgl. hierzu auch Finkenstaedt 1976, pp. 5-8; sowie: llendrickx et al. 2000.171.

${ }^{12} \mathrm{Vgl}$. Hendrickx et alii 2000. p. 184.
}

Ware aber auch in geringer Anzahl noch in Ägypten bis in die Zeit des Alten Reiches bekannt $^{13}$.

Die Black-Topped Gefäße sind zumeist aus Nilton gefertigt und wurden noch vor dem Brand mit Kieselsteinen o.ä. geglättet und poliert. Durch diesen Arbeitsschritt erreichte man die Verdichtung des Tons, so dass eine hochglänzende Oberfläche entstand. Die Gefäße wurden im offenen Feldbrand bei relativ niedrigen Temperaturen von $600-800^{\circ} \mathrm{C}$ gebrannt $^{14}$, wobei die Keramik mit der Öffnung nach unten auf das Brennmaterial gestellt wurde. Die dabei reduzierte Sauerstoffzufuhr erklärt die charakteristische schwarze Färbung des Randes und des Inneren. Wie die experimentelle Archäologie zeigen konnte, handelt es sich um einen einzigen, und nicht wie noch Lucas glaubte, einen zweiphasigen Herstellungs-prozess ${ }^{15}$.

Abgesehen vom ästhetischen Gesichtspunkt kann der Farbunterschied sicherlich aber auch symbolisch verstanden werden. So haben die beiden Farben rot und schwarz im alten Ägypten eine jeweils spezifische Konnotation. Rot wird generell mit Chaos, Wüste und Tod in Zusammenhang gebracht, während die schwarze Farbe für Fruchtbarkeit und Auferstehung, später auch für die Unterwelt stehen $k{ }^{16}{ }^{16}$. So vereint die Black-Topped-Ware möglicherweise den Kontrast zwischen Leben und Tod und kann somit als Ritualkeramik par excellence verstanden werden. Aus dem Kontext der ersten drei Dynastien konnte Sowada zudem einige Gefäße aus verschiedenen Tempeldepots aus Abydos, Hierakonpolis, Tell Ibrahim Awad und Elephantine zusammenstellen. Diese Gefäße entsprechen nicht mehr den aus der prädynastischen Zeit bekannten Formen, sondern sind in Form von $h s$-Vasen hergestellt. Interessant ist zudem, dass auf einigen Stelen der 4. Dyn. aus Giza in den Opferszenen eben diese BlackTopped-Ware erscheint im Zusammen-hang mit

\footnotetext{
${ }^{13} \mathrm{Vgl}$. Sowada 1990, 85-102.

14 Zum Problem der Herstellung von B-Ware: Sowada 1990, 85-102; Ebenso: Hendrickx et al. 2000, 171-187. Vgl. auch: Baba, Saito 2004, 575-589

${ }^{15} \mathrm{Vgl}$. Lucas 1932, 96; bes. auch: Baba. Saito 2004. 577.

${ }^{16}$ Zum Vgl.: Brunner-Traut 1977, 122-127; ebenso:

Wilkinson 1994; Sowada 1990, 102 und Staehelin 2000
} 
Trankritualen ${ }^{17}$. Es ist daher zu überlegen, ob nicht auch die Gefäße aus der Zeit von Naqada III für solche Rituale oder Libationsopfer im Rahmen des Funerärkults genutzt wurden und sich diese Tradition bis ins Alte Reich überliefert hat.

Die Flasche (Inv.-Nr. 121493) gehört einer feiner gebrannten und bearbeiteten Ware an, der von Petrie so bezeichneten P-Ware, der Redpolished-Ware. Auch hier wurde der Ton vor dem Brand mit einem Stein fein geglättet und auf Hochglanz poliert. Der Brennvorgang erfolgte wohl in einem besseren Ofen - möglicherweise nicht mehr im offenen Feld-brand, sodass es nicht zu der charakteristischen Schmauchung des Randes wie bei den zuvor besprochenen Gefäßen kam. Zudem gehört dieser Keramiktyp der jüngeren Phase der Naqada-Kultur, dem Übergang von Stufe Naqada II zu Naqada III an.

Wie bereits betont, stammen alle Gefäße höchstwahrscheinlich vom Antikenmarkt, was eine genaue Verortung nicht ermöglicht. Wenngleich die Flasche einem etwas jünger datierenden Keramiktyp angehört, ist dennoch möglich, dass wir eine Auswahl einiger Grabgefäße vorliegen haben, die zu einem einzigen Grab gehört. Die Black-Topped-Ware ist nämlich, wie bereits Petrie zeigen konnte, durch-aus noch bis in die Zeit von Naqada II in Benutzung gewesen. Stimmt die Vermutung, wären alle hier vorgestellten Gefäße einem Grabkontext zuzuweisen, der in die Zeit um Naqada Ila (ca. 3600 v. Chr.) und nicht viel später datieren würde. Ebenso ist es aber möglich, dass Sutzu die Gefäße einzeln erwarb.

Der englische Archäologe Sir William M. Flinders Petrie (1853-1942) stellte anhand seiner Beobachtungen auf verschiedenen oberägyptischen Gräberfeldern als einer der Ersten eine Typologie der Grabkeramik auf, die er in Klassen/Waren einteilte. Als wichtigstes Gräberfeld gilt hierbei die 1892 gegrabene Nekropole von Naqada ${ }^{18}$, die zugleich eponym für die oberägyptische, chalkolithische Kultur wurde. Der

\footnotetext{
${ }^{17}$ So die Stele der $W p-m-n f r . t-$ heute Berkley Museum 6.19825. Hier sind die in Form der Ikonographie von ḥnk.t - Bier dargestellt. Vgl. Sowada 1990, 100-101.

${ }^{18} \mathrm{Vgl}$. Petrie 1932, 156.
}

$27 \mathrm{~km}$ nördlich von Luxor situierte Fundplatz enthält neben mehreren Nekropolen verschiedener Zeitstufen auch eine Stadt aus der prädynastischen Epoche Ägyptens, sowie einen Tempel des Neuen Reiches. Des Weiteren spielte die Grabung der Nekropolen von Diospolis parva eine entscheidende Rolle bei der Entwicklung seiner Seriation, den so genannten SequenceDates (S.D.) ${ }^{19}$.

Bei dieser Datierungsmethode handelt es sich um eine der relativen Chronologie. ${ }^{20}$ Neben einer groben zeitlichen Einteilung ging es Petrie aber auch um die Ermittlung der Laufzeiten für die einzelnen Objekttypen.

Neben der Datierung und Erstellung einer Keramiktypologie spielte ein weiterer Aspekt in der Arbeit Petries eine entscheidende Rolle. Die in Naqada gefundenen Objekte und Gräber wiesen keinerlei Gemeinsamkeiten mit dem bisher aus Ägypten bekannten Material auf, so dass Petrie in seiner Erstpublikation an eine Invasorengruppe, die New Race dachte, die am Ende der 6. Dynastie nach Ägypten gekommen sei. Hatte man in den bis dato bekannten Nekropolen vorwiegend mit Hieroglyphen beschriebenes Material und in den Fels geschlagene Gräber vorgefunden, lag nun ein Gräberfeld von vielen hunderten in den Sand gegrabenen Gruben vor, die Skelette in Hockerlage bargen. Diese Gräber wiesen in Form und Inventar so viele Gemeinsamkeiten auf, dass Petrie an eine eigene Kulturgruppe dachte ${ }^{21}$. Aufgrund der fehlenden Überschneidungen, die einen Vergleich mit anderen Gräberfeldem hätten ermöglichen können, vermutete er, dass es keinen Kontakt der hiesigen Bevölkerung mit den Ägyptern gegeben habe. Zudem wurden anthropologische Messungen an den Schädeln

\footnotetext{
${ }^{19}$ Erstmals wurden sie von Petrie 1899 vorgelegt und späler in der Publikation über Diospolis parva erweitert. $\mathrm{Vgl}$. Petrie 1899, 295-301; Petric 1901, bes. pl. II. Auch von mathematisch-statistischer Seite wurde das Problem bereits hinterfragt: Vgl. Kendall 1963, 657-680; ebenso: Kendall 1970. 125-135.

${ }^{20} \mathrm{Vgl}$. Petrie 1899, 295.

21 Petrie, Quibell 1896, 59. Sie bezeichnen die Einwanderer als Zugehörige eines Volkes.
} 
durchgeführt ${ }^{22}$ und über die Vergleiche mit algerischen Populationen der Schluss gezogen, dass die hier bestattete Bevölkerungsgruppe aus den Oasen gekommen sein musste ${ }^{23}$. Darauf haben sie die ägyptische Bevölkerung der thebanischen Ebene vernichtet und sich schließlich in diesem Raum angesiedelt. Sie knüpften sodann Fernhandelsverbindungen bis nach Palästina und Syrien, wo sich ein ähnliches Fundgut im archäologischen Befund abzeichnet. Aufgrund stratigrafischer Beobachtungen und Vergleiche mit den bereits bekannten Nekropolen des Neuen- und Mittleren Reiches, schlussfolgerten die Ausgräber Petrie und Quibell, dass die Invasion der New Race im Übergang der 6.-11. Dynastie ${ }^{24}$ (ca. 2170-2020 v. Chr.) erfolgt sein müsste - eine Zeit, die aufgrund der inschriftlichen Belege mit chaotischen Zuständen in Verbindung gebracht wurde. Erst der Widersacher Petries, Jacques de Morgan, der ein Jahr später in Naqada eine große Mastaba der 1. Dynastie ausgrub und auch hieroglyphische Inschriften fand, ordnete das Gräberfeld richtig in die ägyptische Prähistorie ein ${ }^{25}$.

Wenngleich die kulturhistorischen Theorien Petries keinen Bestand haben, ist seine Keramiktypologie bis heute Grundlage jeglicher Beschäftigung mit keramischem Material aus der formativen Phase Ägyptens und soll im Folgenden nochmals kurz skizziert und mit der nötigen Kritik versehen werden. Sein System lässt sich auf drei Arbeitsschritte zurückführen: 1) Das Erstellen des Korpus, für den die Objekte nach Materialgattung getrennt in neun Klassen, je mit einem Großbuchstaben benannt, eingeteilt wurden (vgl. auch Tabelle 1). Die Unter-kategorien erhielten eine Zahl: z.B. B 16. Ins-gesamt kam Petrie damit auf insgesamt 700 Einzeltypen bzw. -formen ${ }^{26}$.

\footnotetext{
${ }^{22}$ Anthropologisch wurden von 3000 Bestattungen allein 301 Schädel im University College bearbeitet: Vgl. Fawcett. Lee $1901,408-467$.

23 Petrie, Quibell 1896.63-64. Diese Theorie wurde auch von Derry 1956 aulgenommen und versucht mit Schädelmaßen erneut zu untermaucrn. Vgl. Derry 1956, 83. Dic Theorie findet sich ebenso noch bei Emery 1964. 3435. Erst dic neucre Forscherung ist von dieser InvasorenTheoric abgekommen.

2:1 Petric. Quibell 1896.61

25 De Morgan 1897. 15.

${ }^{20} \mathrm{Vgl}$. Ilendrickx 2006,61
}

In einem zweiten Schritt konnte mithilfe des Corpus nun im Feld praktisch gearbeitet werden. D.h. Petrie ließ nicht von jedem neu gefundenen Objekt eine Zeichnung anfertigen, sondern beschränkte sich darauf, nur noch die Korpus-Nummer des jeweiligen Stückes zu vermerken. Dies sparte freilich erheblich $\mathrm{Zeit}^{27}$, sagt aber auch Einiges über die Qualität seiner späteren Publikationen aus. Diese Nummern wurden auf einer so genannten Grabkarte, einer Karteikarte, bestehend aus neun Spalten, aufgeschrieben, sodass man einen klaren Überblick über die Typen und Typenvergesellschaftungen in den Gräbern erhielt. Um die einzelnen Bestattungen datieren zu können, musste Petrie die einzelnen Grabkarten sortieren. Dabei fanden drei Nekropolen Berücksichtigung: Naqada, Ballas und Diospolis parva ${ }^{28}$. Betrachtet wurden allerdings nur die Gräber, die wenigstens fünf oder mehr verschiedene Keramikgefäße aufwiesen. Schlussendlich kam er also auf eine Summe von 900 Grabkarten, die geordnet werden mussten. $\mathrm{Zu}$ beachten ist, dass in seinem System letztlich weniger als ein Viertel aller ausgegrabenen Bestattungen Berücksichtigung fanden.

In einem dritten und letzten Schritt wurden nun die chronologischen Betrachtungen und Merkmale gegeneinander abgewogen. Dabei stützte Petrie sich auf die stratigrafischen Beobachtungen, die Entwicklung der einzelnen Klassenformen (besonders anhand der Wellenhenkelgefäße [Wavy-handled-Ware]), das Erstellen von Gruppen aufgrund von Ähnlichkeiten, stilistische Merkmale und die jeweilige Laufzeit jedes einzelnen Typs.

Wichtig für die Gesamtdatierung und das Sortieren der Karten waren die Wellenhenkelgefäße. Petrie versuchte mithilfe von typologischen Betrachtungsweisen eine Entwicklung dieses Keramikgefäßtypus aufzustellen. Dabei ging er davon aus, dass die Stücke mit den plastisch geformten Seitenhenkeln die älteren und die mit den aufgemalten Ornamentmustern, die typologisch jüngeren Objekte seien. Letzteres nennen wir heute typologisches Rudiment. Auch

\footnotetext{
${ }^{27}$ Petric 1932, 157.

28 Hendrickx 2006, 61.
} 
wenn diese Betrachtungen durchaus kritisch als Evolutionismus ausgelegt werden können und Petrie keinerlei Hinweise darauf hatte, dass es sich wirklich so verhielt - denn es hätte ja ebenso anders herum sein können - behielt er mehr oder weniger Recht. Die Grabung von de Morgan zeigte, dass Gefäße mit aufgemaltem Wellenhenkel zusammen mit Schriftdokumenten auftraten, also typologisch wirklich den jüngeren Typ darstellen.

Im Folgenden untersuchte Petrie die Gräber der anderen Keramikwaren, die zu-sammen mit W-Ware auftraten ${ }^{29}$. Er bemerkte, dass vor allem die C-Ware (White-Cross-Lined) nur mit frühen W-Gefäßen zusammen vor-kamen, also die CWare einem älteren Typus zu-gehörig war. Das Zusammenvorkommen von $\mathrm{C}$-Ware und anderen Waren lieferte weitere relativ-chronologische Ansätze. So wurde schließlich mit allen Waren verfahren. Dabei spielen laut Petrie, der das weitere Vorgehen selbst nicht näher kommentiert, statistische Erwägungen eine Rolle ${ }^{30}$. Dies führte auch zu mehrmaliger Umstellung der verschiedenen Typen, wie er eindrücklich darlegt $^{31}$.

Alle 900 Karten konnte Petrie schließlich in 50 Gruppen mit je 18 Gräbern unterteilen, die dann nach ihren Formen vom jüngsten zum ältesten geordnet wurden. Sie bilden die so genannten S.D. - Sequenzdaten oder „Staffeldaten". Die 50 Gräbergruppen unterteilte er dabei nochmals in sieben stages (Stadien), da es zwischen den einzelnen Staffeldaten auch Überschneidungen gab, also ein Gefäßtypus mehreren Staffeldaten zugeordnet werden konnte:
a. 30
b. $31-34$
c. $35-42$
d. $43-50$
e. $51-62$
f. $63-71$
g. $72-80$

Diese Stadien wurden wiederum drei Zeitphasen untergeordnet. Dabei wurden die S.D. von 30-80 vergeben. Insofern zeigte Petrie sich

\footnotetext{
${ }^{29} \mathrm{Vgl}$. Petrie 1901, 6.

${ }^{30}$ Petrie $1901,8$.

${ }^{31}$ Petrie 1901.8.
}

äußerst vorausschauend, indem er die S.D. 1-29 für noch ältere Kulturkomplexe und die Daten $81-100$ für spätere freilie $\beta^{32}$.

Mit der Festsetzung der Datenbasis für die Keramik konnte Petrie die anderen Objektklassen wie Kupfergeräte, Schminkpaletten, Steingefäße u.a. in die S.D. einordnen. Dabei listete er die Gefäßklassen und -formen, sowie ihr Vorkommen mit den jeweiligen Objekten auf, und gliederte sie so in sein System ein. Die Staffeldaten wurden zudem in drei Zeitstufen unterteilt, die Petrie allesamt nach den Fundorten Amratian (el-Amrah), Gerzean (el-Gerza) und Semainean (Semaina) benannte.

Die S.D., dass war Petrie allerdings auch bewusst, hatten freilich keinen genauen Aussagewert über die Laufzeit einer jeweiligen Epoche. Naturwissenschaftliche Datierungsmethoden existierten noch nicht und so setzte Petrie vorerst eine Laufzeit von 1.000-2.000 Jahren für den gesamten S.D.-Komplex fest. ${ }^{33}$ Später revidierte er die Aussage und unterteilte die Zeiten wie folgt ${ }^{34}$ :
a) S.D. 48-40: 2-3 Generationen
b) S.D. 40-44: 4-5 Generationen
c) S.D. 74-78: 60 Jahre

Erst 1931 führte Alexander Scharff, ein Kritiker Petries, die heute so geläufige Terminologie von Negade I-II ein, die dann 1957 von Werner Kaiser auf die Naqada I-III-Zeit erweitert wurde und der damit eine Überarbeitung des Petrie-Systems vornahm.

Der Versuch, die S.D. auch in absolute Daten zu überführen, scheiterte bei Petrie. Denn hier ging er nicht von statistischen Daten und klaren Ideen aus, sondern ließ sich von seiner Phantasie treiben. So versuchte er mit alten Annalen und überlieferten Listen den Beginn für die 1. Dyn. auf 5500 v. Chr., den Anfang seiner Naqada-Gräber gar mit 8000 v. Chr. festzulegen. Dieser Chronologie ist aber bereits zu seinen Lebzeiten heftigst widersprochen worden. ${ }^{35}$

\footnotetext{
${ }^{32} \mathrm{Vgl}$. Petrie 1899, 299.

${ }^{33}$ Petrie 1901, 8.

${ }^{34}$ Nach Petrie 1901, 29 und 45.

${ }^{35} \mathrm{Vgl}$. Peet 1922. 6 sq.
} 
Die ${ }^{14} \mathrm{C}$-Methode wurde erstmals von Libby 1955 in Saqqara angewendet. ${ }^{36}$ Später untersuchte er mit seiner Methode auch Haut- und Haarmaterial, das in der Nekropole von Naqada geborgen wurde und publizierte erste ${ }^{14} \mathrm{C}$-Daten für Ägypten, darunter auch einige Gräber aus Naqada. Anhand des Haut- und Haarmaterials, was sich recht gut erhalten hatte, kam er schließlich auf die begehrten absoluten Daten, auch wenn diese noch unkalibriert waren ${ }^{37}$.

Wenngleich die Seriation einen bedeutenden Fortschritt für die relative Chronologie des frühen Ägypten darstellt, erfuhr sie bereits zu Petries Zeiten Kritik ${ }^{38}$. Und selbst Petrie musste einsehen, dass sein System nur bedingt funktionierte. So bereitete z.B. die Einordnung der etwas älter datierenden Badari-Kultur mit S.D. 21-37 einige Schwierigkeiten. Denn diese Gräber waren ausgesprochen fundarm und wiesen kaum mehr als 1-2 Gefäße als Beigaben auf. Für eine ordentliche Seriation benötigte man aber Gräber mit mindestens fünf verschiedenen Gefäßtypen. Doch dies traf nicht nur auf die Badari-Kultur $\mathrm{zu}^{39}$, auch das Amratian war nur schwer mit den von Petrie kalkulierten S.D. zu erfassen. Zudem kritisierte Scharff die schematische Arbeitsweise ${ }^{40}$, da von einem Typ jeweils nur eine Zeichnung existierte, und bei den anderen Publikationen nur noch die Typenbezeichnung genannt wird, und sich so nur schwer Keramikvergleiche machen ließen. Dies hatte zur Konsequenz, dass auf Feinheiten, die möglicherweise aufgrund von Regionalismen bestanden haben könnten, nicht beachtet wurden $^{41}$. Hauptkritikpunkt war aber vor allem die Datierung der W-Ware, die nämlich laut Scharff nicht bereits in S.D. 35, sondern viel später in S.D. 43 auftaucht.

Zusammenfassend lässt sich feststellen, dass die von Petrie aufgestellte Typologie nicht

\footnotetext{
${ }^{36} \mathrm{Vgl}$. Libby 1955. Eine gute Zusammenfassung bietet Rachet 1977, 110.

${ }^{37} \mathrm{Vgl}$. Hendrickx 1999, 13-81.

${ }^{38}$ Kritiker waren u.a. Scharff 1926; Peet 1922 und Legge 1913.101-113

i9 Man muss allerdings zugeben, dass es bis heute nicht gelungen ist. trotz großer Bemühungen. die Badari-Kultur besser zu gliedern. Zum neuesten Versuch vgl. Math 2007 , $205-219$.

to Scharff 1926, 16.

4l Scharif 1926.73.
}

auf jedes frühzeitliche Material anwendbar ist, was vor allem auf die Gräber mit weniger als fünf verschiedenen Keramiktypen zutrifft und auch die Einordnung der Badari-Kultur ein-schließt. Zudem muss festgehalten werden, dass die Typologie sich auf allein 900 Gräber von 4.000 stützt, also nur einen kleinen Teil der ge-samten Befunde widerspiegelt. Ein weiteres Problem ist die Abgrenzung der Typologie von der Chronologie $^{42}$. Insgesamt handelt es sich also um ein sehr evolutionistisches System, was am besten bei der von Petrie aufgestellten Ent-wicklung der Wellenhenkelgefäße zum Aus-druck kommt.

$\mathrm{Zu}$ beachten sind zudem, wie bereits von Scharff völlig zu Recht herausgestellt, die regionalen Unterschiede, die sich auch in der Herstellung der einzelnen Keramiktypen und formen aufzeigt. Petrie hatte vorwiegend oberägyptisches Material bearbeitet, das, wie sich gezeigt hat, durchaus einige Unterschiede zum unterägyptischen Fundspektrum aufweist.

Dennoch soll bei aller Kritik nicht vergessen werden, dass es sich um den ersten relativchronologischen Datierungsansatz han-delt, der sich um die Keramik bemüht und der bis heute im Großen und Ganzen Verwendung findet und mit der nötigen kritischen Betrachtung und Abänderung aufgrund neuer Grabungsergebnisse weiterhin große Bedeutung hat (vgl. auch Tabelle 2).

\footnotetext{
${ }^{42}$ Hendrickx 2006, 63.
} 


\begin{tabular}{|l|l|l|l|l|l|}
\hline Abk. & Klasse & S.D. laut Petrie & Untersucht nach & Zusammenhang & Tonart \\
\hline B & Black-Topped & $34-35$ & Brenntechnologie & & Nilton \\
\hline P & Red-Polished & 39 & Bearbeitungsspuren & & Nilton \\
\hline F & Fancy & $31-70$ & Form & $\begin{array}{l}\text { meist red- } \\
\text { polished }\end{array}$ & $\begin{array}{l}\text { meist } \\
\text { Nilton }\end{array}$ \\
\hline C & $\begin{array}{l}\text { White-Cross- } \\
\text { Lined }\end{array}$ & & Dekoration & Red-polished & Nilton \\
\hline N & Incised Black & $46-47$ & Dekoration & & Nilton \\
\hline D & Decorated & $46-47$ & Dekoration & meist Late & Mergelton \\
\hline W & Wavy-Handled & & Morph. Details & & Mergelton \\
\hline R & Rough Ware & 43 & $\begin{array}{l}\text { Tonart/Bearbeitungsspur } \\
\text { en }\end{array}$ & & Nilton \\
\hline L & Late & $68-69$ & Tonart/Chronologie & einige Rough & $\begin{array}{l}\text { meist } \\
\text { Mergelton }\end{array}$ \\
\hline
\end{tabular}

Tabelle 1: Die einzelnen Warenklassen nach Petrie 1920, Corpus.

\begin{tabular}{|l|l|l|l|l|l|}
\hline $\begin{array}{l}\text { Petrie }^{44}, \\
\text { Brunton }^{45}, \\
\text { Vandier }^{46}\end{array}$ & S.D. & $\begin{array}{l}\text { Scharff }^{47}, \text { Kantor }^{48}, \\
\text { Baumgarte }^{49}\end{array}$ & Kaiser $^{50}$ & Hendrickx $^{51}$ & Hassan $^{52}$ \\
\hline Semainean & $\begin{array}{l}61-75 \\
{[65-76]^{53}}\end{array}$ & Protodynastic & Naqada IIIa-d & $\begin{array}{l}\text { Naqada } \\
\text { IIIA-D }\end{array}$ & $\begin{array}{l}\text { Terminal } \\
\text { Predynastic }\end{array}$ \\
\hline Gerzean & $38-60$ & Naqada II & Naqada IIa-d & Naqada IIC-D & $\begin{array}{l}\text { Late } \\
\text { Predynastic }\end{array}$ \\
\hline Amratian & $30-37$ & Naqada I & Naqada Ia-c & Naqada I-IIB & $\begin{array}{l}\text { Middle } \\
\text { Predynastic }\end{array}$ \\
\hline Badarian & $21-29$ & Badarian & Badari & Badari & $\begin{array}{l}\text { Early } \\
\text { Predynastic }\end{array}$ \\
\hline
\end{tabular}

Tabelle 2: Zusammenstellung der Terminologie der einzelnen Bearbeiter.

${ }^{43}$ So bei Petrie 1901. 12; vgl. auch Petrie 1921.

${ }^{41}$ Petric 1920, 46-50.

t5 Vgl. Brunton 1937: ebenso: Brunton 1948.

${ }^{46} \mathrm{Vgl}$. Vandier 1953.

${ }^{47} \mathrm{Vgl}$. Scharff 1931, 16-30.

${ }^{48}$ Kantor 1944. 110-136.

49 $\mathrm{Vgl}$. Baumgartel 1970.

${ }^{50} \mathrm{Vgl}$. Kaiser 1957.

"Hendrickx 1999; Hendrickx 2006, 55-93.

${ }_{52}^{5} \mathrm{Vgl}$. Hassan 1980, 203-207. Ebenso: Hassan 1984, 681-683.

${ }^{53}$ Später abgeänder: cf. Friedman 1994, 42. 


\section{TÖNERNER GRABKONUS}

Inv.-Nr. 121494 (Fig. 1, 3-4)

Bei diesem Objekt handelt es sich um einen $22,5 \mathrm{~cm}$ langen und 7,5-7,9 $\mathrm{cm}$ breiten Konus aus Nilton mit Stempelsiegelabdruck. Der Körper ist länglich nach unten spitz zulaufend gestaltet und handgefertigt, sodass er mehrere Fingerabdrücke aufweist. Es handelt sich um im erhabenen Relief herausgedrückte Hieroglyphen, die in vier vertikalen Kolumnen angeordnet sind:

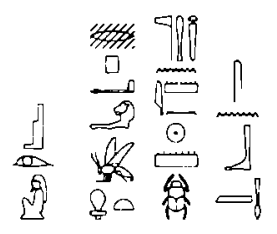

$\mathrm{Zu}$ lesen ist also:

\begin{tabular}{|c|c|}
\hline$[/] W \zeta J R$ & OSIRIS, \\
\hline [2] $\quad(r)-p^{r}, I$ & Graf, Bürgermeister \\
\hline$h l m \cdot \mathbf{k}^{\prime}-b j t j$ & Siegelbewahrer des Königs, \\
\hline [.3] hm-ntr tpj $n \mathrm{Jmm}$ & Hohepriester des \\
\hline$M n-h l p r-R^{r}$ & Men-Cheper-Ra- \\
\hline [4] $s n b m, e-h r w$ & $\begin{array}{l}\text { snb - gerechtfertigt } \\
\text { Stimme. }\end{array}$ \\
\hline
\end{tabular}

Das Stempelsiegel selbst ist länglich oval und wurde mit Hilfe einer negativ eingeschnittenen Vorlage in dem noch feuchten Tonkonus gedrückt, der dann bei relativ geringer Temperatur gebrannt worden ist. Der Ton ist mineralisch und organisch gemagert und enthält einen recht hohen Anteil an Glimmer. Die Oberfläche ist punktuell mit einer dünnen Salzschicht überzogen, ansonsten in tadellosem Zustand.

Auf dem Objekt befindet sich der Name

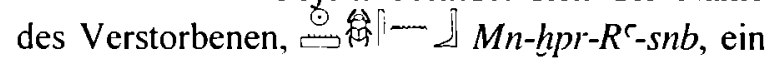
basilophor gebildeter Privatname, der mit „Thutmosis III. ist gesund ${ }^{54 ،}$ übersetzt werden kann. Die Titulatur bestätigt zudem, dass es sich um einen Hohepriester des Amun handelt ${ }^{55}$. Wenngleich wir vor dem selben Problem stehen, wie wir es bereits für die Objekte der formativen Phase konstatieren müssen, also weder Provenienz noch andere weiterführende Informationen zu diesem Stück vorliegen haben, kann bereits Einiges über die Herkunft des Konus ver-

\footnotetext{
${ }^{54} \mathrm{Vgl}$. Ranke 1935. 150,15.

55 Dieser Titel taucht erstmals in der 18. Dyn. auf. Vgl. Wörterbuch der ägyptischen Sprache III, 1971, 89, III.
}

mutet werden. Der Titel „Hohepriester des Amun" ist erstmals unter Ahmose (1550-1525 v. Chr.) im Neuen Reich (ca. 1550-1069 v. Chr.) belegt und bezeichnet das höchste Priesteramt dieser Epoche mit Sitz im oberägyptischen Theben $^{56}$. Dies lässt erwarten, dass auch unser Grabkegel aus diesem Gebiet stammt. Zudem ist zu bemerken, dass ein Großteil der ca. 400 bisher bekannten Sets von Grabkegeln im Raum Theben gefunden worden ist. Der basilophor ge-bildete Name kann zudem nicht vor Herrschafts-antritt von Thutmosis III., also ca. 1486 v. Chr., auftauchen, sodass er uns einen terminus post quem liefert. Tatsächlich ist uns eine Person mit dem oben genannten Namen aus dem Raum Theben bekannt, der das Amt des Oberpriesters des Amun während der Regierungszeit des Pharao Thutmosis III. (1486-1425 v. Chr.) in der 18. Dynastie innehatte. Er ist wohl der Besitzer von zwei sich in Sheikh Abd el-Qurna (Theben West) befindlichen Privatgräbern: Grab TT 86 und Grab TT $112^{57}$. Mittlerweile wird allerdings von verschiedenen Seiten die Meinung vertreten, es habe zwei Personen des gleichen Namens gegeben, wobei möglicherweise $M n-h p r-R^{c}-s n b$ (II.) der Neffe des ersten ist und in dem wohl etwas jünger datierenden Grab TT 112 bei-gesetzt wurde $^{58}$. Unter anderem befinden sich im University College, London sechs Grabkegel ${ }^{59}$ mit vergleichbarer Inschrift mit der Herkunftsangabe TT 86. Dies macht es sehr wahrscheinlich, dass auch das Exemplar, dass sich in Bukarest befindet, zu diesem Ensemble gehört und somit aus TT 86 stammt.

Grabkoni tauchen erstmals in der 11. Dyn. in ägyptischen Gräbern auf und sind anfänglich

\footnotetext{
${ }^{56}$ Zur Geschichte und Entwicklung des Titels im Laufe der ägyptischen Geschichte: Bierbrier 1977, 1241-1249.

${ }^{57}$ Vgl. Porter, Moss 1960, 175 - ein Plan des Grabes befindet sich auf 176. Vgl. zur Person ebenso: Lefebvre 1929, 233-235. \$8. Sehr kurz: Helck 1982, 43.

$58 \mathrm{Vgl}$. hierzu Bryan 2000, 244. Ähnlich wird von Cline, O'Connor 2006, 108-109 argumentiert.

${ }_{59}$ Es handelt sich um die folgenden Objekte: UC 37578, UC 37579, UC 37580; UC 37581; UC 37582; UC 37583. Als Herkunftsangabe wird zudem TT 86 angegeben. Auf der Internetseite des Instituts sind auch unter www.digitalegypt.ucl.ac.uk die Photographien der einzelnen Objekte abrufbar. Bereits Lefebvre 1929, 234 hat auf weitere 185 Grabkoni aus dem Grab berichtet. Weitere Objekte befinden sich heute u.a. in Hildesheim, Zagreb und im Louvre. Vgl. hierzu auch: Cihó 1983, 43.
} 
noch unbeschriftet. ${ }^{60}$ Einige dieser frühen Koni sind aber teilweise bemalt und mit Symbolen wie Kreuzen verziert. ${ }^{61}$ Erst im Zuge des Neuen Reiches befinden sich Name und Titel des jeweiligen Grabinhabers auf den Objekten. Zudem können sie kurze Gebete und Segenswünsche, sowie die Namen anderer Angehörige wie die der Ehefrau enthalten. ${ }^{62}$ Einige der Stücke weisen Reste von Bemalung auf, zumeist rote oder blaue ${ }^{63}$ Tünchung, wie sie sich aber an unserem Objekt nicht bestätigen lassen. Während bis dato ca. 400 Sets solcher Koni be-kannt sind, stammen nur 80 Objekte aus in situ dokumentierten Befunden. ${ }^{64}$ Sie befinden sich zumeist in zwei bis vier Reihen übereinander in der weißgetünchten Lehmziegelgrabfassade eingemauert, was ihnen auch den Namen "Friesziegel" eingebracht hat. ${ }^{65}$ Sie bilden somit ein Schmuckband unterhalb der Hohlkehle bzw. des Pyramidions, wie wir es von den thebanischen Privatgräbern kennen. ${ }^{66}$ Auf diese Nutzung weist auch die Großzahl der gefundenen Objekte hin, die sich im Vorhof der Grabanlage fanden, somit von der Grabfassade abgestürzt bzw. herausgebrochen sind. ${ }^{67}$

Neben den vielen archäologischen Nachweisen existieren aber auch einige gemalte Szenen auf Grabwänden des Neuen Reiches, die die oben genannte Nutzung der Grabkegel unterstützen. Eine interessante Darstellung findet sich ab der Ramessidenzeit in den thebanischen Privatgräbern. Auf einigen dieser Szenen, wie wir sie im Grab des Nfr-Htp (TT 49), einem Oberschreiber des Amuntempels am Ende der 18. Dynastie, oder im Grab des Vierten Amun-

\footnotetext{
${ }^{60}$ So wurden beispielsweise einige Exemplare von Winlock in einem Grab nahe von Deir el-Bahari 1927 entdeckt: Vgl. Winlock 1928. 5-6.

${ }^{\circ} \mathrm{Vgl}$. ebenso BorchardL Königsberger, Ricke 1934, 35.

${ }^{62} \mathrm{Vgl}$. Eggebrecht 1977, 858.

${ }^{63}$ Dic blaue Tünchung ist allerdings äußerst selten. Vgl. Borchardt, Königsberger, Ricke 1934, 33. Sie be-ziehen sich auf eine Aussage von G. Daressy.

64 www.digitalegypt.ucl.ac.uk: Solche in-Situ-Befunde finden sich auch bei Rhind 1862. 136-137.

${ }_{65} \mathrm{Vgl}$. Borchardt, Königsberger, Ricke 1934, 25.

${ }^{60}$ So bereits von Borchardt, Königsberger, Ricke 1934, 28 festgestellt - erneut Eggebrecht 1977, 858.

67 Hierauf macht auch schon Guksch 1997, 9-13 aufmerksam.
}

priesters Raa-iaA aus der 19. Dynastie finden, hat bereits Borchardt hingewiesen (vgl. Taf. $1,4-5){ }^{68}$

Grabkegel sind in Ägypten bis in die 26. Dynastie belegt, wobei sie aber nicht nur inschriftlich, sondem auch in der Form und Größe Veränderungen erfahren. So sind die ersten, noch unbeschrifteten Koni aus der 11. Dynastie bis zu $52,5 \mathrm{~cm}$ lang, was einer ägyptischen Elle entspricht. Sie werden im Laufe ihrer Entwicklung jedoch immer kleiner. In der Spätzeit schließlich erreichen sie nur noch $1 / 3$ bis $1 / 4$ ihrer anfänglichen Größe. ${ }^{69}$ Eine weitere Besonderheit ist die regionale Beschränkung ihres Vorkommens. Während wir die Objektkategorie aus dem Mittleren Reich relativ weitgestreut aus ElRizeikat $^{70}$, Armant ${ }^{71}$, Naqada ${ }^{72}$ und Abydos ${ }^{73}$ kennen, stammen nahezu alle Objekte des Neuen Reiches aus der Umgebung von Theben oder Aniba $^{74}$. Auch die spätzeitlichen Belege kommen aus der Region um Theben, was generell mit der Problematik des Archaismus in Verbindung gebracht werden kann ${ }^{75}$.

Die Bedeutung der Grabkegel ist bis heute weitgehend ungeklärt. In Zusammenhang mit den mesopotamischen Friesziegeln hat man auch die ägyptischen zunächst als bloße Dekorationselemente angesehen ${ }^{76}$. Meinungen, es handele sich um Scheinbrote, Pflastersteine oder Weiterentwicklungen von Mumienetiketten wurden ebenso diskutiert. Dabei hat gerade die Theorie die Scheinbrote betreffend vor allem durch die Form der Koni Einiges für sich. Die zumeist runde Form der Stempelfläche lässt Eggebrecht vermuten, es könnte sich um eine Form von Sonnensymbolik handeln, wobei der

\footnotetext{
${ }^{68}$ Zum Vgl.: Borchardt 1934, 28 und Abb. 1; ebenso Guksch 1997, 12. Weitere Gräber mit ähnlichen Szenen sind TT 178 (Grab des $K n-R^{2}$ ), Zeit Ramses II.) und das Grab TT 181 (Gravierer Ipwky, Zeit Amenhotep III.).

${ }^{69}$ Arnold 1972, 19 sowie Taf. $13 \mathrm{~d}$.

${ }^{70}$ Daressy 1926, 18-19.

${ }^{71}$ Mond, Myers 1937, pl. 22,6.

${ }^{72}$ Arnold 1968, 35 mit Anm. 2.

${ }^{73}$ Daressy 1926, 19

$74 \mathrm{Vgl}$. Eggebrecht 1977, 858. Weitere Stücke stammen möglicherweise aus Naga ed-Dêr. Vgl. von Bissing, 1926. 171 .

${ }^{75}$ Zum Problem des Archaismus: generell Brunner 1970 , 151-161; ebenso: Brunner 1975, 386-395. In Verbindung mit Theben nun auch Kahl 1999.

${ }^{76} \mathrm{Vgl}$. Borchardt 1934, 34.
} 
Tote durch die Schreibung des Namens in den nehmen könnte. sonnenförmigen Konus am Sonnenlauf teil-

\section{LITERATUR}

Arnold 1968 - D. Arnold, Bemerkungen zu den Gräbern in El-Târif, MDAIK, 23, 1968, p. 25-35.

Arnold 1972 - D. Amold, Bericht über die vom Deutschen Archäologischen Insitut Kairo im Mentuhotep-Tempel und in El-Târif unternommenen Arbeiten, MDAIK, 28, 1972, p. 13-31.

Baba, Saito 2004 - M. Baba, M. Saito, Experimental Studies on the Firing Methods of Black-Topped Pottery in Predynastic Egypt. In: K.M. Ciałowicz et al. (Hrsg.), Egypt at its Origins, Orientalia Lovaniensia Analecta, 172, Leuven, 2004, p. 575-589.

Baumgartel 1970 - E. J. Baumgartel, Petrie's Naqada Exvavation. A Supplement, London, 1970.

Bierbrier 1977 - M. L. Bierbrier, Hohepriester des Amun. In: LÄ, II, 1977, p. 1241-1249.

Borchardt 1899 - L. Borchardt, Pflastersteine, ZÄS, 37, 1899, p. 80-81.

Borchardt, Königsberger, Ricke 1934 - L. Borchardt, O. Königsberger, H. Ricke, Friesziegel in Grabbauten, ZÄS, 70, 1934, p. 25-35.

Brunner 1970 - H. Brunner, Zum Verständnis der archaisierenden Tendenzen in der ägyptischen Spätzeit, Saeculum, 21, 2/3, 1970, p. 151-161.

Brunner 1975 - H. Brunner, Archaismus. In: LÄ, I, 1975, p. 386-395.

Brunner-Traut 1977 - E. Brunner-Traut, Farbsymbolik. In: LÄ, II, 1977, p. 122-127.

Brunton 1937 - G. Brunton, Mostagedda and the Tasian Culture, London, 1937.

Brunton 1948 - G. Brunton, Matmar. The British Museum Expedition to Middle Egypt 1929-1931, London, 1948.

Bryan 2000 - B. Bryan, The $18^{\text {th }}$ Dynasty Before the Amarna Period. In: I. Shaw (Hrsg.), The Oxford History of ancient Egypt, Oxford, 2000, p. 218-271.

Buchez 2007 - N. Buchez, Chronologie et transformations structurelles de l'habitat au cours du prédynastique. Apport des mobiliers céramiques funéraires et domestiques du site d'Adaima (Haute-Egypte), unpubl. Diss., Toulouse, 2007.

Cihó 1983 - M. Cihó, Two Unpublished XVIII' Dynasty Objects from Rumania, GöttMisz, 69, 1983, p. 17-22.

Cihó 1984 - M. Cihó, The Egyptian Collection of the Faculty of History and Philosophy-Bucarest, GöttMisz, 71, 1984 , p. 83-87.

Cihó et aliii 1988 - M. Cihó et alii, Antichităti egiptene in colecffile din România, Cluj-Napoca, 1988.

Clinc, O'Connor 2006 - E. H. Cline, D. O'Connor (Hrsg.), Thutmose III. A New Biography, Ann Arbor, 2006.

Daressy 1926 - G. Daressy, Le voyage d'inspection de M. Grébaut en 1889, ASAE, 26, 1926, pp. 18-19.

Davies, Macadam 1957 - N. de G. Davies, M. F. L. Macadam, A Corpus of inscribed Egyptian funerary cones, Oxford, 1957.

De Morgan 1897 - J. de Morgan, Recherches sur les Origines de l'Egypte. Ethnographie préhistorique et tombeau royal de Négadah, Paris, 1897.

Derry 1956 - D. Derry, The Dynastic Race, JEA, 42, 1956, p. 80-85.

Eggebrecht 1977 - A. Eggebrecht, Grabkegel. In: LÄ, II, 1977, p. 857-859.

Emery 1964 - W. B. Emery, Ägypten. Geschichte und Kultur der Frühzeit 3200-2800 v. Chr., München, 1964.

Fawcett, Lee 1901 - C.D. Fawcett, A. Lee, A Second study of the Variation and Correlation of the Human Skull, with special Reference to the Naqada Crania, Biometrika. A journal fort he statistical study of Biological problems, 1, Cambridge, 1901, p. 408-467.

Finkenstaedt 1976 - E. Finkenstaedt, The Chronology of Egyptian Predynastic Black-Topped Ware, ZÄS, 103, 1976, p. 5-8.

Franke 1984 - D. Franke, Probleme der Arbeit mit altägyptischen Titeln des Mittleren Reiches, GöttMisz, 83, 1984 , p. $103-124$.

Friedman 1994 - R. F. Friedman, Predynastic Settlement Ceramics of Upper Egypt: A comparative Study of the Ceramics of Hemamieh, Nagada, and Hierakonpolis, Berkley, 1994. 
Guksch 1997 - H. Guksch, Die Grabkegelaufschrift Davis-Macadam Nr. 475 - und ein Ende! GöttMisz, 158, 1997, p. 9-13.

Hartmann 2008 - R. Hartmann, The Chronology of Naqada I tombs in the Predynastic Cemetery $U$ at Abydos. In: R. Friedman, L. McNamara (Hrsg.), Abstracts of papers presented at the Third International Colloquium on Predynastic and Early Dynastic Egypt, London, 2008, p. 103-104.

Hassan 1980 - F. A. Hassan, Radiocarbon Chronology of Archaic Egypt, AncNearEastSt, 39,3, 1980, p. $203-207$.

Hassan 1984 - F. A. Hassan, Radiocarbon Chronology of Predynastic Nagada Settlements, Upper Egypt, Current Anthropology, 25, Chicago, 1984, 5, p. 681-683.

Helck 1982 - W. Helck, Mencheperreseneb. In: LÄ, IV, 1982, p. 43.

Hendrickx 1999 - S. Hendrickx, La chronologie de la préhistoire tardive et des débuts de l'histoire de l'Egypte, Archéo-Nil, 9, 1999, p. 13-81.

Hendrickx et al. 2000 - S. Hendrickx/R. Friedman/F. Loyens, Experimental Archaeology concerning BlackTopped-Pottery from Ancient Egypt and the Sudan, CahCerEg, 6, 2000, p. 171-187.

Hendrickx 2002 - S. Hendrickx et al., Un cimetière particulier de la deuxième dynastie à Elkab, Archéo-Nil, 12, 2002 , p. $47-54$.

Hendrickx 2006 - S. Hendrickx, Predynastic - Early Dynastic Chronology. In: E. Hornung, R. Krauss, D. A. Warburton (Hrgs.), Ancient Egyptian Chronology. Handb. der Orientalistik I, 83, Leiden-Boston, 2006, p. 55-93.

Hoffman 1980 - M. A. Hoffman, Egypt Before the Pharaohs: the Prehistoric Foundations of Egyptian Civilization, Austin, 1980.

Ioniţă 2004 - M. Ioniţă, La aniversară. File din istoria inceputurilor Muzeului National din București, MuzNaț, XVI, 2004, p. 195-215.

Kahl 1999 - J. Kahl, Siut-Theben: Zur Wertschätzung von Traditionen im alten Ägypten, Probleme der Ägyptologie, 13, Leiden-Boston, 1999.

Kaiser 1957 - W. Kaiser, Zur inneren Chronologie der Naqadakultur, AGeo, August 1957, p. 69-77.

Kantor 1944 - H. J. Kantor, The Final Phase of Predynastic Culture, Gerzean of Semainean? JNES, 3, 1944, p. $110-136$.

Kendall 1963 - D. G. Kendall, A Statistical Approach to Flinders Petrie's Sequence-Dating, Bull. Internat. Statistical. Inst., 40,1963 , p. $657-680$

Kendall 1970 - D. G. Kendall, A Mathematical Approach to Seriation, Philosophical Transaction of the Royal Society A (Mathematical, Physical \& Engineering Sciences), 269, 1970, p. 125-135.

Lefebvre 1929 - G. Lefebvre, Histoire des grands prêtres d'Amon de Karnak jusqu'à la Xxf́ dynastie, Paris, 1929.

Legge 1913 - G.F. Legge, New Light on Sequence-dating, Proceedings of the Society of Biblical Archaeology, London, 35, 1913, p. 101-113.

Libby 1955 - W.F. Libby, Radiocarbon Dates, Chicago, 1955.

Lucas 1932 - A. Lucas, Black and Black-Topped Pottery, ASAE, 32, 1932, p. 96.

Garis-Davies 1957 - N. de Garis Davies, A Corpus of Inscribed Egyptian Funerary Cones, Oxford, 1957.

Math 2007 - N. Math, Eine innere Chronologie der Badari-Kultur? Möglichkeiten und Aspekte, ÄgLev, 27, 2007, p. 205-219.

Mond/Myers 1937 - R. Mond/O.H. Myers, Cemeteries of Armant I, Egyptian Exploration Society, 42, London, 1937.

Peet 1922 - T. E. Peet, The Antiquity of Egyptian Civilization being a plea for some attempt to formulate the laws which should form the basis of archaeological argument, JEA, 8, 1922, p. 5-12.

Petrie 1899 - W.M.F. Petrie, Sequences in Prehistoric Remain, Journal Royal Anthropological Institute Great Britain, 29, 1899, p. 295-301.

Petrie 1901 - W. M. F. Petrie, Diospolis parva. The cemeteries of Abadiyeh and Hu I898-1899, Egipt Exploration Fund, 20, London, 1901.

Petrie 1920 - W. M. F. Petrie, Prehistoric Egypt, British School of Archaeology in Egypt, 23, London, 1920.

Petric 1921 - W. M. F. Petrie, Corpus of Prehistoric Pottery and Palettes, British School of Archaeology in Egypt, 24, London, 1921.

Petrie 1932 - W. M. F. Petrie, Seventy Years in Archaeology, London, 1932.

Petrie, Quibell 1896 - W.M.F. Petrie, J.E. Quibell, Naqada and Ballas, London, 1896. 
Porter, Moss 1960 - B. Porter, R. Moss, Topographical Bibliography of Ancient Egyptian Hieroglyphical Texts, Reliefs and Paintings I. The Theban Necropolis, Oxford, 1960.

Potra 1944 - G. Potra, Cezar Bolliac numismat şi arheolog. Cronica numismatică şi arheologică. Foaie de informaţii a Societăţii Numismatice Române, XVIII, 130, 1944, p. 243.

Rachet 1977 - G. Rachet, Universul arheologiei. Tehnică, istorie, bilant, Bucureşti, 1977.

Ranke 1935 - H. Ranke, Die ägyptischen Personennamen, I, Glücksstadt, 1935.

Rhind 1862 - A. H. Rhind, Thebes, its tombs and their tenants, London, 1862.

Scharff 1926 - A. Scharff, Das vorgeschichtliche Gräberfeld von Abusir el-Meleq, Leipzig, 1926.

Scharff 1931 - A. Scharff, Die Altertïmer der Vor- und Frühzeit Ägyptens, Mitt. aus der ägypt. Sammlung. Staatl. Mus. Berlin 5, 1931.

Sowada 1990 - K. N. Sowada, Black-topped Ware in Early Dynastic Contexts, JEA, 85, 1990, p. 85-102.

Staehelin 2000 - E. Staehelin, Von der Farbigkeit Ägyptens, Leipzig, 2000.

Vandier 1953 - J. Vandier, Manuel d'Archéologie égyptienne l: Les époques de formation. La Préhistoire et les trois premières dynasties, Paris, 1953.

Vianu 1992 - M. A. Vianu, Mihail C. Sutzu, colectionar, StCercIstorV, 43, 1992, 2, p. 127-137.

von Bissing 1926 - F. von Bissing, 'Grabkegel' in Naga ed Deir, ActaOr, 5,2, 1926, p. 171-173.

Wilkinson 1994 - R. Wilkinson, Symbol and Magic in Egyptian Art, London, 1994.

Winlock 1928 - H. Winlock, The Egyptian Expedition 1925-1927, BMetrMus, February 1928, p. 5-6.

ROBERT KUHN

Ägyptologisches Institut/Ägyptisches Museum der Universität Leipzig - Georg Steindorff

Goethestr. 2, 04109, Leipzig, Deutschland

Rkuhn18@aol.com 


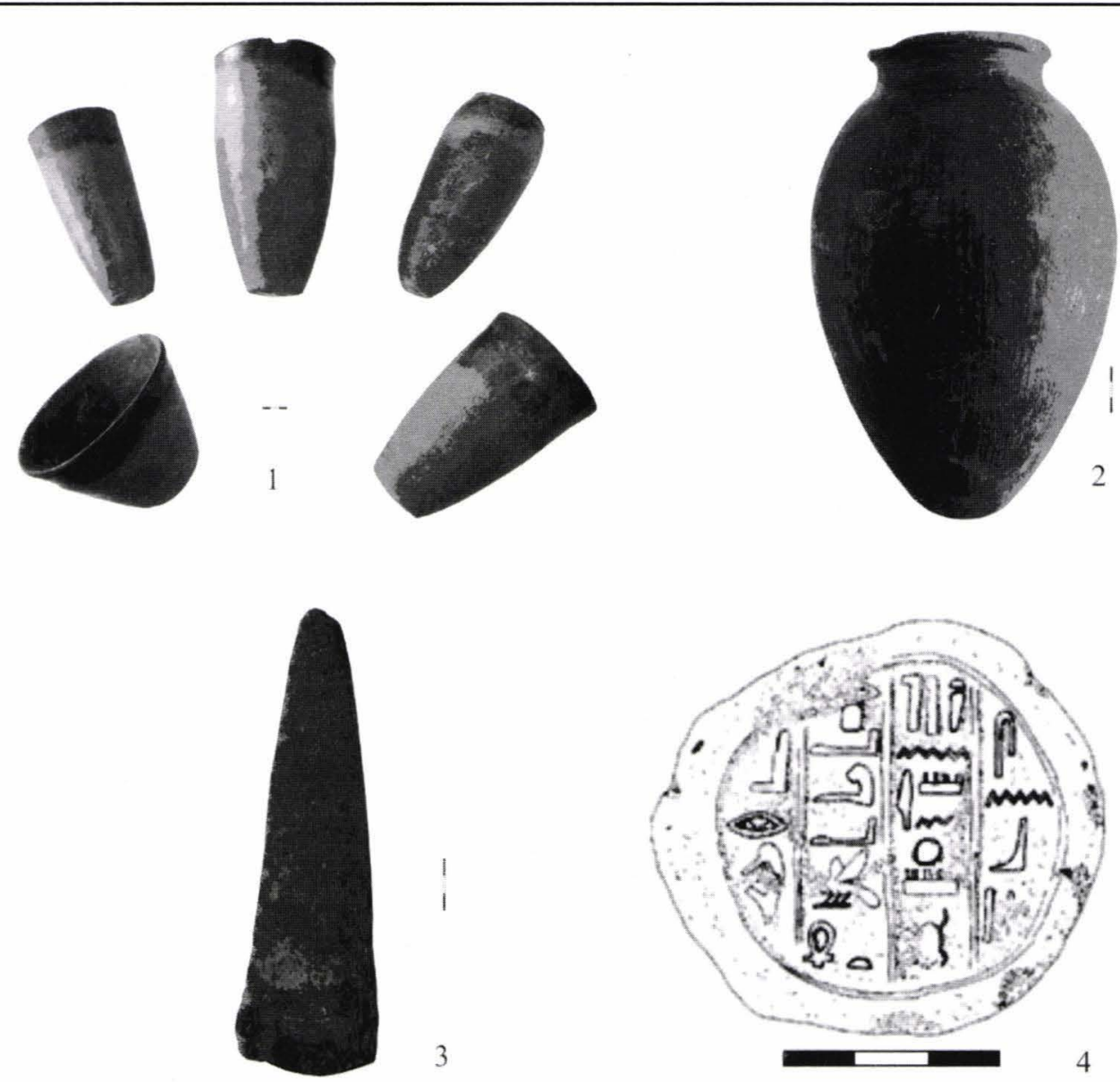

3

4
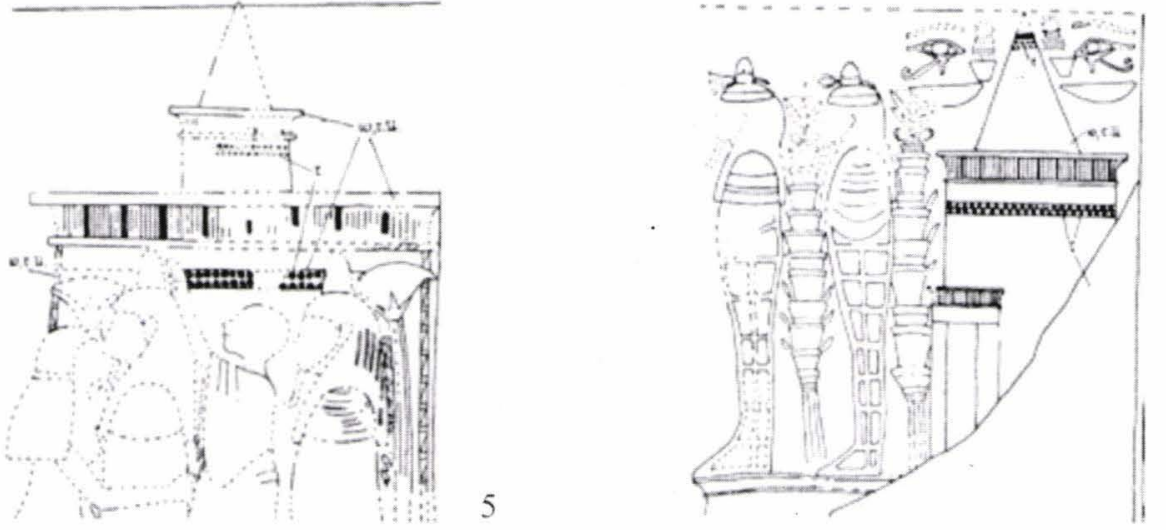

6

PI. 1 - 1. Ensemble der Black-Topped-Ware aus der Privatsammlung Sutzu, heute MNIR Inv.-Nr.n: 121488-121491 und MNIR Inv.-Nr. 121495 (Fotos: R.K.); 2. Red-Polished-Vase, MNIR Inv.-Nr. 121493; ehemals Privatsammlung Sutzu (Foto: R.K.);

3/4. Grabkonus aus Grab TT 86, MNIR Inv.-Nr. 121494, ehemals Privatsammlung Sutzu - Zeichnung des Siegelstempels auf dem Konus (R.K.); 5. Friesziegel im Grab des Nefer-Hotep (TT 49), Ende 18. Dyn. (nach Borchardt et al. 1934, p. 28);

6. Friesziegel im Grab des Raa-iaA (TT 159), 19. Dyn. (nach Borchardt et al.1934, p. 28) 

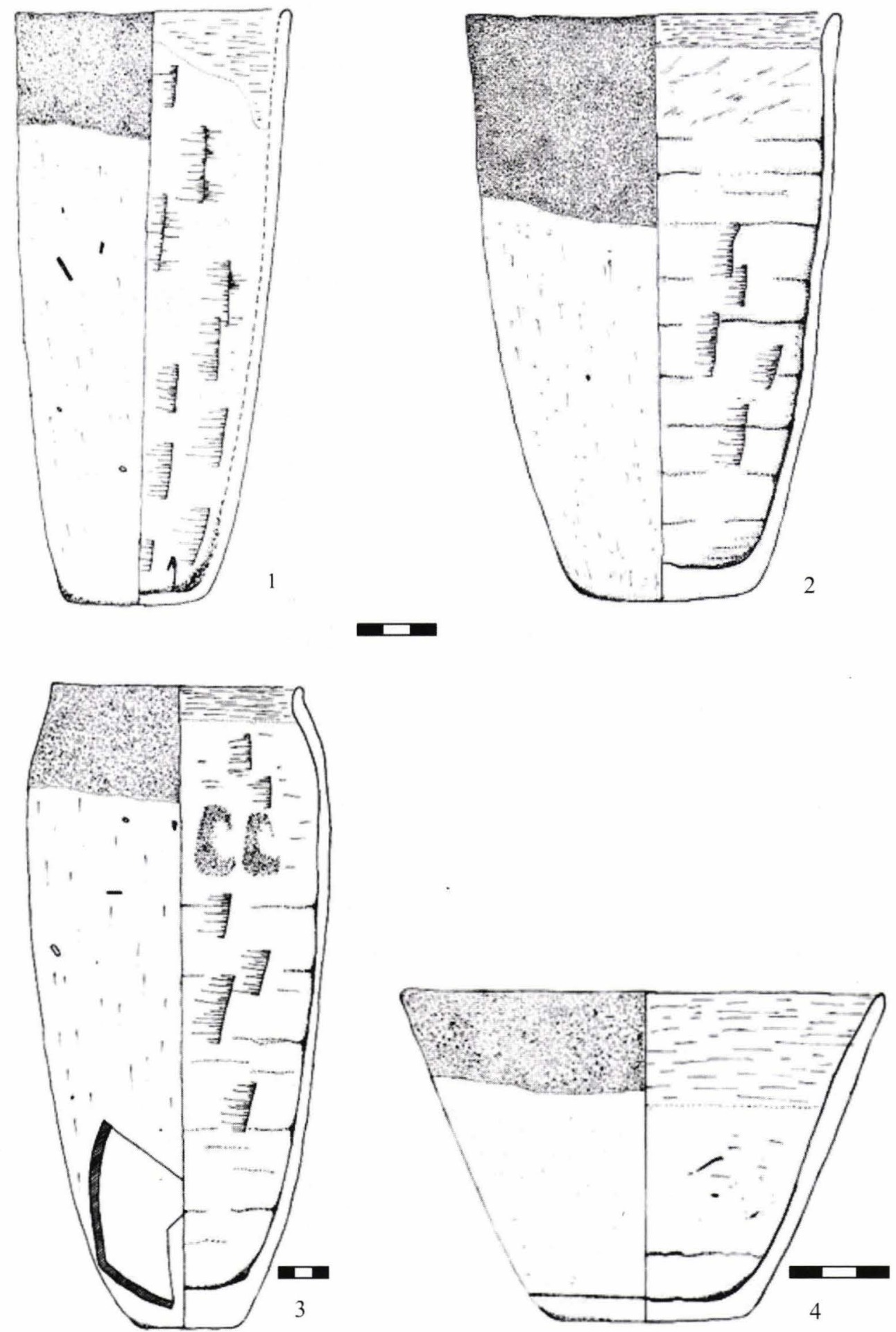

PI. 2 - 1. B-Ware; MNIR Inv.-Nr. 121490/alt E. 78; 2. B-Ware; MNIR Inv.-Nr. 121489; 3. B-Ware; MNIR Inv.-Nr. 121495; 4. B-Ware; MNIR Inv.-Nr. 121488. Alle Zeichnungen R.K. 

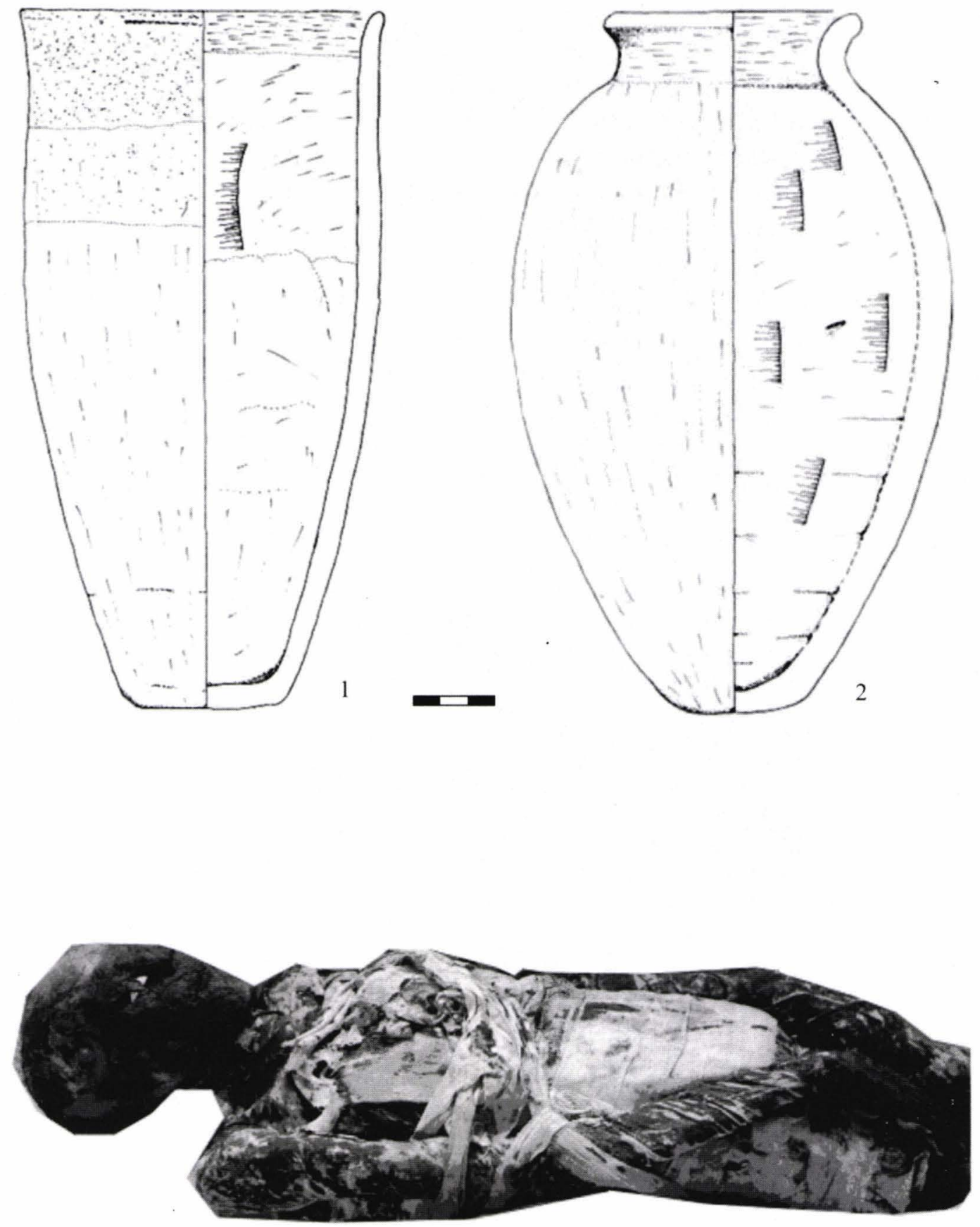

PI. 3 - 1. B-Ware; MNIR Inv.-Nr. 121491; 2. P-Ware, MNIR Inv.-Nr. 121493; 3. Mumie des Bes, ehemals Privatsammlung Bolliac. Alle Abb.: R.K. 

STUDII PLURIDISCIPLINARE 
